Biedermann Andrea Regina (Orcid ID: 0000-0001-9819-6969)

\title{
Magnetic pore fabrics: the role of shape and distribution anisotropy in defining the magnetic anisotropy of ferrofluid-impregnated samples
}

\section{Andrea R. Biedermann ${ }^{1}$}

${ }^{1}$ Institute of Geological Sciences, University of Bern, Baltzerstrasse 1+3, 3012 Bern, Switzerland

Corresponding author: Andrea Biedermann (andrea.regina.biedermann@gmail.com)

\section{Key Points:}

- Magnetic anisotropy of ferrofluid-impregnated rocks is a promising tool to assess the pore fabric

- The average pore shape, arrangement of pores with respect to each other, and ferrofluid susceptibility all affect the measured anisotropy

- Models quantitatively predict the expected anisotropy for simple pore shapes and arrangements$$
\text { (at }
$$<smiles>C1=CC2CC1C2</smiles>
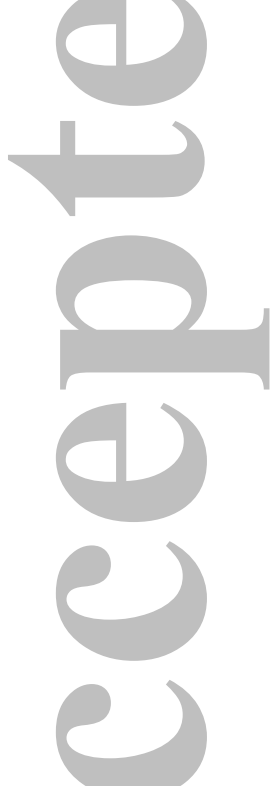

This article has been accepted for publication and undergone full peer review but has not been through the copyediting, typesetting, pagination and proofreading process which may lead to differences between this version and the Version of Record. Please cite this article as doi: 10.1029/2019GC008563 


\begin{abstract}
Pore fabrics define physical properties of a rock, such as permeability and elasticity, both of which are important to many geological, hydrological and environmental applications. Minerals and hence pores are often preferentially aligned, leading to anisotropy of physical properties and preferred flow directions. Preferred flow paths are defined by the shape and arrangement of pores, and a characterization of this pore fabric forms the basis for prediction of fluid flow directions. Magnetic pore fabrics (MPF), i.e., magnetic anisotropy measurements on ferrofluid-impregnated samples, are a promising and fast way to characterize the pore fabric of connected pores in $3 \mathrm{D}$, while analysing a large number of pores with sizes down to $10 \mathrm{~nm}$, without the need for any a priori knowledge about fabric orientation. Empirical relationships suggest that MPF is related to the pore shape and orientation and approximates permeability anisotropy. This study uses models including shape and distribution anisotropy to better understand and quantify MPF, using simple pore shapes and pore assemblies measured in previous studies. The results obtained in this study show that (1) shape anisotropy reliably predicts the MPF of single pores, (2) both shape and distribution anisotropy are needed to predict MPF of pore assemblies, and (3) the anisotropy parameters $\mathrm{P}, \mathrm{L}$, and $\mathrm{F}$ are affected by the intrinsic susceptibility of the ferrofluid in addition to pore geometry. These findings can help explain some of the variability in empirical relationships, and are an important step towards a quantitative understanding and application of MPF in/geological and environmental studies.
\end{abstract}

\title{
Plain Language Summary
}

To produce clean drinking water, use geothermal energy or control contamination, it is necessary to understand how fluids flow underground. They have to find their way from pore to pore. As soon as pores are elongated or flattened, fluids can move more easily and thus faster in some directions than others. It is desirable to predict such preferred flow directions, and a good description of the pore space is needed to do so. Many methods exist to characterize the pore space, and one of these is based on the directional dependence of magnetic properties of samples, whose pores have been filled with strongly magnetic fluid. The method is efficient and promising, but unfortunately it is not well understood how the magnetic data reflects the details of the pore space. The models developed here help define and quantify the factors controlling the observable magnetic properties. This understanding will make the method more applicable and useful in geothermal, hydrological and environmental applications. 


\section{Introduction}

The pore fabric, i.e., the shape, arrangement, and connectivity of pores, defines many physical properties of a rock, including its permeability and elastic properties [Bear, 2013; Dullien, 1992; Schön, 2015]. When rocks display a preferred mineral or grain alignment, e.g. as a consequence of sedimentary transport or subsequent deformation, this alignment results in elongated pores with a shape preferred orientation. This pore fabric in turn is reflected by anisotropy of physical properties, e.g. permeability, conductivity or seismic velocity [Bear et al., 1987; Beard and Weyl, 1973; Jones and Meredith, 1998; Rasolofosaon and Zinszner, 2002; Weger et al., 2009]. Permeability is important in geological, hydrological and environmental applications, e.g. (1) geothermal energy production, (2) migration of groundwater and hydrocarbon, and (3) leakage and transport of contaminants. The anisotropy of permeability, in particular, defines preferred flow and transport directions, and thus helps constrain the location of drill holes for geothermal doublets, or predict the spread of contamination after a leakage.

For all these predictions, it is necessary to understand the pore fabric, and hence the fluid flow on a pore-scale. A systematic characterization and description of the pores and their relationship to each other is needed. Mineral grains in rocks have different sizes, sorting degree and roundness, leading to complex pore structures with pore sizes varying over several orders of magnitude, with a large range of shapes and arrangements, making them challenging to describe [Bear, 2013; Bennett et al., 1989; Dullien, 1992]. Nevertheless, the importance of pore fabrics led to the use of numerous methods to describe pore fabrics directly (e.g. optical and electron microscopy, and X-ray computed tomography (XRCT)), or indirectly through the anisotropy they cause in rock properties (permeability anisotropy, seismic anisotropy, magnetic pore fabrics). Direct methods have the advantage that pores can be mapped in either 2D (microscopy) or 3D (XRCT), and therefore, their spatial, size, and orientation distributions can be evaluated. A major limitation of these direct methods is their resolution, i.e. small pores cannot be imaged well. Additionally, the 2D surfaces used for microscopy may not be representative, and sample preparation may alter the pores at the observation surface. Finally, these methods are time-consuming and especially for XRCT require a large amount of data storage space and processing time. Indirect methods provide a time- and cost-effective way to determine the average 3D pore orientation, allowing to analyse large sample sets to assess e.g. small-scale or regional-scale variations in pore fabrics. Indirect methods target a large number of pores of all sizes. They do not provide information on individual pores or size distributions, which are often not needed in fluid flow applications. Permeability anisotropy is the most direct measure of flow properties. However, in practice permeability is often measured along 3 directions in 3 different samples, insufficient to define the full second-order tensor that describes permeability, and prone to artefacts if permeability varies between samples. Elastic properties are sensitive to pores and their orientation, but cannot distinguish between connected and isolated pores, and are also affected by mineral texture, grain boundaries, and cracks. Being a $4^{\text {th }}$-order tensor, elastic properties are also more complicated than permeability anisotropy. Hence, they are not ideal for assessing pore fabrics in fluid flow studies [Almqvist et al., 2011].

Anisotropy of magnetic susceptibility (AMS) of ferrofluid-impregnated samples has been proposed as a fast and efficient method to assess the average 3D structure of connected pores [Parés et al., 2016; Pfleiderer and Halls, 1990; 1994]. Magnetic pore fabric (MPF) measurements can capture pores down to $10 \mathrm{~nm}$ [Parés et al., 2016; Robion et al., 2014], and because AMS characterizes the full $2^{\text {nd }}$ order tensor, no a priori knowledge on the fabric orientation is necessary. The few studies that exist on MPF found promising empirical relationships between the average pore orientation and the AMS of ferrofluid-impregnated 
samples [Jones et al., 2006; Pfleiderer and Halls, 1990; 1993], or between permeability anisotropy and MPF [Benson et al., 2003; Hailwood et al., 1999; Louis et al., 2005;

Pfleiderer and Halls, 1994] (Figure 1). Pfleiderer and Halls [1990] state that the 'AMS is related inversely to the demagnetizing factor, which itself relates inversely to axial ratios of pores', so that the MPF is a direct reflection of the pore shape. Based on this, Hrouda et al. [2000] developed the equivalent pore concept (EPC), a method to quantify the average pore shape based on the MPF. Jones et al. [2006] found that the EPC underestimated the axial ratio of pores in their synthetic samples, particularly at low ferrofluid concentration, and (1) proposed a correction factor, and (2) suggested to use fluid with high susceptibility. In contrast, diluted ferrofluid (e.g., 1:5 or 1:100) has been used to avoid susceptibilities above the instrumental measurement limit [Benson et al., 2003; Parés et al., 2016]. Most studies do not specify the concentration or intrinsic susceptibility of the ferrofluid used. Inconsistencies and variability in the empirical relationships between (1) pore shape and preferred pore orientation and MPF orientation, as well as (2) the axial ratio of the average pore and the degree of MPF have been observed [Esteban et al., 2006; Nabawy et al., 2009; Pfleiderer and Halls, 1994] (Figure 1). This indicates that the relationship between pore fabric and related AMS is not as straightforward as initially proposed, and more work is needed to fully understand the origin of the MPF.

AMS has been used for decades as a fast and efficient proxy for rock textures, and hence tectonic and geodynamic processes [Borradaile and Henry, 1997; Borradaile and Jackson, 2010; Hrouda, 1982; Martín-Hernández et al., 2004; Owens and Bamford, 1976; Tarling and Hrouda, 1993]. This experience in interpreting magnetic fabric data can perhaps help lead the community towards an improved understanding of MPF. Similar to MPF, magnetic fabrics on non-impregnated rocks were long interpreted based on empirical relationships for fabric orientation or fabric strength [Balsley and Buddington, 1960; Hirt et al., 1988; Kligfield et al., 1977; Kneen, 1976]. Also similar to MPF, exceptions to those empirical relationships have been observed; e.g. different correlations exist between AMS orientation and flow direction in lava [Khan, 1962; Wing-Fatt and Stacey, 1966], and certain minerals or grain sizes produce 'inverse' or 'anomalous' fabrics [Borradaile et al., 1993; Rochette, 1988; Rochette et al., 1999]. Careful and systematic investigations of factors contributing to anisotropy (magnetocrystalline, shape and distribution anisotropy) [Graham, 1954; Grégoire et al., 1995; Hargraves, 1959; Hargraves et al., 1991; Mainprice and Humbert, 1994; Stephenson, 1994] together with the characterization of single crystal properties [Biedermann, 2018, and references therein] now allows one to model and understand even these 'anomalous' and complex fabrics [Biedermann et al., 2018].

Analogously, a more systematic and quantitative understanding of MPF needs to be developed, partly based on the recent develops in AMS interpretation. One difference between MPF and grain anisotropy is that pores are connected; therefore, rather than measuring the anisotropy of a series of magnetic grains, the anisotropy of a large 3D construct of magnetic material is characterized. As an additional complication, rocks often contain magnetic grains which could potentially interact with the particles in the ferrofluid, thus leading to formation of clusters.

Whereas the magnetic analysis of pore fabrics has high potential in that large numbers of samples could be characterised in an efficient way, the details of how MPFs originate are poorly understood. This study aims to contribute to a better understanding of MPF by investigating the influence of shape and distribution anisotropy for simple pore shapes and assemblies of pores, and for different ferrofluid susceptibilities. The model developed here can later be adapted to more complicated 3D pore structures in rocks that contain magnetic grains, and may lay the foundation for a more reliable and quantitative interpretation of MPF in the future. 


\section{Theory}

Analysis of MPF is based on the assumption that the ferrofluid homogeneously fills all connected pores in the sample. The large susceptibility contrast between ferrofluid and rock leads to self-demagnetization, which results in shape anisotropy as soon as pores are nonspherical. Hence, the AMS measured on a ferrofluid-impregnated sample is thought to be related to the average pore shape [Hrouda et al., 2000; Pfleiderer and Halls, 1990].

Self-demagnetization occurs when a strongly magnetic body (e.g., grain or ferrofluidfilled pore) is surrounded by less magnetic material, reducing the apparent susceptibility and magnetization of the body. This magnetostatic effect is closely related to body shape. It is weakest along the longest axis of the grain, and strongest along its short axis, resulting in a maximum susceptibility along the long axis and minimum along the short axis [Clark, 2014; Clark and Emerson, 1999; Lowrie, 1997]. This geometry-dependence is represented by the demagnetization tensor $N$, which can be determined exactly for ellipsoidal shapes [Osborn, 1945; Stoner, 1945]. Approximations exist for other bodies, e.g. cylinders [Sato and Ishii, 1989]. The observed (apparent) susceptibility of a strongly magnetic body is $k_{\text {obs }}=$ $\left(I+k_{\text {int }} N\right)^{-1} k_{\text {int }}$, where $k_{\text {int }}$ is the intrinsic susceptibility, and $I$ a unit matrix [Clark, 2014]. In magnetic anomaly studies, self-demagnetization becomes important for intrinsic susceptibilities $>0.1(\mathrm{SI})$, and crucial for $k_{\text {int }}>0.5$. For very large susceptibilities, $k_{o b s}$ can be approximated by $N^{-1}$ [Abbott et al., 2007]. Shape anisotropy has long been used to characterize the shape preferred orientation of magnetite grains in rocks [Archanjo et al., 1995; Grégoire et al., 1998; Parry, 1965; Salazar et al., 2016; Trindade et al., 1999].

Similarly, it is expected to reflect the preferred orientation of ferrofluid-filled pores [Hrouda et al., 2000; Jones et al., 2006; Pfleiderer and Halls, 1990]. Ferrofluid susceptibilities reach up to 4 (SI), and thus, self-demagnetization remains important even when they are diluted.

A second effect that may be important for MPF is distribution anisotropy.

Magnetostatic interactions between strongly magnetic isotropic grains lead to an increased susceptibility along the long axis of a linear arrangement, or a minimum susceptibility normal to a planar arrangement [Hargraves et al., 1991; Muxworthy and Williams, 2004; Stephenson, 1994]. Analogously, a non-uniform spatial distribution of ferrofluid-filled pores may lead to distribution anisotropy. On the micro-scale, MPFs could be viewed as distribution anisotropy of spherical particles, whose non-uniform spatial distribution is controlled by the geometry of the pore space. In any case, distribution anisotropy can be calculated via the secondary field generated by any particle on its neighboring particles, affecting the effective field experienced by each particle: $\vec{H}_{e f f}=\vec{H}_{e x t}+\sum_{i=1}^{n} \vec{h}_{i}$, where $\vec{H}_{\text {ext }}$ is the external field, $n$ the number of particles, and $\vec{h}_{i}$ the secondary field generated by the magnetization of particle $i$ [Stephenson, 1994]. The secondary field can be calculated from the dipole formula, and has a radial component $h_{r}=(2 m \cos (\theta)) /\left(4 \pi r^{3}\right)$, and tangential component $h_{\theta}=(m \sin (\theta)) /$ $\left(4 \pi r^{3}\right)$, where $m$ is the magnetic moment, $m=M^{*} V$, with $M$ the magnetization and $V$ the volume of the pore or grain, $r$ the distance between pores, and $\theta$ the angle between the magnetic moment vector and the distance vector. The effective field for each particle, and for the entire population, is an anisotropic function of the applied external field, and this is essentially the source of the distribution anisotropy.

Whereas solely shape anisotropy is considered in the EPC model, both shape anisotropy (related to the shape of each individual pore) and distribution anisotropy (related to pore arrangement, or the distribution of magnetic particles within the ferrofluid) may be important for MPF. The relative contribution of shape anisotropy and distribution anisotropy in rocks is under debate, and depends on the details of the grain arrangement and the 
magnetic properties and shape of individual grains [Cañón-Tapia, 2001]. Grégoire et al. [1995] studied synthetic samples made of magnetite-rich and magnetite-free layers, and concluded that distribution anisotropy contributes largely to the fabric of magnetite-bearing rocks, as long as magnetite forms clusters. These results are supported by synthetic models showing that the magnetic fabric of arrays of ellipsoidal particles is dominated by the shape of the array rather than individual particle geometries [Cañón-Tapia, 1996]. Conversely, Grégoire et al. [1998] found that shape anisotropy in granitic rocks is related to the shape of magnetite grains, rather than their distribution, and Gaillot et al. [2006] claim that effects of magnetic interactions on AMS are overestimated in theoretical models. Only one study discussed the potential influence of distribution anisotropy on MPF. Jones et al. [2006] examined a stack of oblate cylinders (crack-like fabric), a plane of prolate cylinders (capillary-type fabric), and a line of prolate cylinders next to each other (bedding fabric). The MPF of the first two was attributed to the shapes of the individual voids, whereas the latter was explained by the spatial distribution of the cylinders. This would mean that some of their MPF measurements were defined by shape anisotropy and the other by distribution anisotropy. Determining the relative contribution of shape and distribution anisotropy to a measured MPF is an important goal and requires additional research. It is noteworthy that the influence of distribution anisotropy may explain some of the variability found in empirical relationships between MPF and pore shapes.

\section{Methods}

\subsection{Model setup}

Models were developed to compute shape and distribution anisotropies, for the simplified void geometries of previously measured synthetic samples [Jones et al., 2006; Pfleiderer and Halls, 1990] (Figure 2). These geometries were chosen so that the modeling results can be directly compared with published experimental data. Both studies had used synthetic samples with single or multiple pores. Pfleiderer and Halls [1990] provide dimensions for single pores, but not for pores in multiple-pore samples. Therefore, only the former will be used here. Jones et al. [2006] used pore arrangements resembling bedding-, capillary- and crack-like fabrics. They do provide the dimensions of each pore in their multipore samples, but not the distances between them. The spheroidal pores in that study are described solely by their aspect ratios, and the dimensions presented here are arbitrary numbers corresponding to those. Note that the models approximate pores with ellipsoids or cylinders, whereas the pore shapes in the synthetic samples may have deviated from these idealized geometries. For example, the oblate and prolate pores in Jones et al. [2006] were approximated with half-spheres glued together at different distances, i.e., they are not exactly spheroidal. Nevertheless, ellipsoids approximate those pore shapes within a few percent [Jones et al., 2006].

Because these synthetic pores are large (on the order of $\mathrm{mm}$ ) compared to the magnetic particles in the ferrofluid (on the order of $10 \mathrm{~s}$ of $\mathrm{nm}$ ), it is reasonable to model their MPF using a homogeneous fluid of constant susceptibility. Note that for the smaller pores found in rocks, models based on distributed particles may be more appropriate. Models were computed for $k_{\text {int }}$ corresponding to the fluid used for the experiment, if known, or for a range of susceptibilities if the exact value was not provided in the experiment description. Jones et al. [2006] impregnated their samples with an oil-based ferrofluid, EMG905 at 50\% concentration, representing an intrinsic susceptibility of 1.09 SI. Additional experiments with ferrofluid concentrations of 5\%,10\%, 20\%, 50\% and $100 \%$ were performed on the spheroidal sample with axial to radial dimension of 1.2 . For $100 \%$ concentration, the intrinsic susceptibility is given as 3.34 SI, but the susceptibilities for the lower concentrations are not 
provided. Pfleiderer and Halls [1990] used the water-based ferrofluid EMG705, which, according to its technical specifications (ferrotec.com) possesses an intrinsic susceptibility of 4.04 SI. If diluted ferrofluid was used, the susceptibility would be lower. To investigate variations in MPF with $k_{\text {int }}$, a range of susceptibilities between 0.1 and 4.04 SI will be used.

The calculated magnetic properties are represented by the eigenvalues $\left(k_{1} \geq k_{2} \geq k_{3}\right)$ and eigenvectors of the susceptibility tensor, and the degree $\left(P=k_{1} / k_{3}\right)$ and shape of the anisotropy $\left(U=\left(2 k_{2}-k_{1}-k_{3}\right) /\left(k_{1}-k_{3}\right)\right)$ [Jelinek, 1981]. The anisotropy degree $P$ equals 1 when susceptibility is isotropic (i.e., $k_{1}=k_{2}=k_{3}$ ), and increases as anisotropy becomes more pronounced. The shape parameter $U$ varies from -1 for rotationally prolate ellipsoids $\left(k_{1}>k_{2}=k_{3}\right)$ to +1 for rotationally oblate ellipsoids $\left(k_{1}=k_{2}>k_{3}\right)$. In addition to these parameters, published experimental data were also presented as magnetic lineation $L=k_{1} / k_{2}$ and foliation $F=k_{2} / k_{3}$, which will be used here to directly compare the model results with the experiments. The magnetic lineation $L$ is 1 when $k_{1}=k_{2}$ (rotationally oblate ellipsoid) and increases as the ratio of the maximum and intermediate susceptibilities increases. Analogously, the magnetic foliation $F=1$ when $k_{2}=k_{3}$ (rotationally prolate ellipsoid). Calculated and measured properties were compared in terms of the orientation of principal directions, and the degree and shape of the anisotropy, represented by the parameters, $P, L, F$ and $U$.

\subsection{Shape anisotropy models for single pores}

Every pore can possess shape anisotropy, whether or not the sample contains only a single void, or an assembly of voids. For each pore, demagnetization tensors were computed for the bodies best approximating its shape for which demagnetization factors are defined, i.e., ellipsoids and cylinders [Osborn, 1945; Sato and Ishii, 1989]. The observed susceptibility tensor was then calculated from these demagnetization tensors and the (possible range of) intrinsic susceptibility(ies) used in the published experimental studies [Jones et al., 2006; Pfleiderer and Halls, 1990].

MPF models based on shape anisotropy were compared to experimental data for both samples with a single pore, and those containing multiple pores. For samples made up of assemblies of equal pores, the measured AMS should correspond to that of a single pore if the average pore shape is solely responsible for the MPF as predicted by the EPC model. Conversely, differences in modeled and measured results indicates that interactions between pores are important.

\subsection{Shape and distribution anisotropy models for pore assemblies}

Other than single pores, pores forming part of an assembly may possess distribution anisotropy in addition to shape anisotropy. Distribution anisotropy was computed from the secondary fields generated by each pore on its neighbors, assuming infinite lines or planes of equal pores.

A first set of models includes spherical, oblate and prolate ellipsoidal pores with their unique axis parallel to $\mathrm{z}$, aligned along to the $\mathrm{x}-, \mathrm{y}-$, and $\mathrm{z}$-axes, and in planes parallel to $\mathrm{xy}$, $\mathrm{yz}$, and zx (Figure 3). The aspect ratios of the pores correspond to the spheroidal samples of Jones et al. [2006]. Because the magnetic force of a dipole decays inversely with distancecubed, magnetostatic interactions were computed for nearest neighbors only. Due to symmetry, the magnetic properties of pores in $\mathrm{x}$ - and $\mathrm{y}$-lines, or in yz and zx planes are expected to be equal, but different from the properties of z-lines and xy planes. Pore separation (centre-to-centre distance) was varied from pore diameter (i.e., neighboring pores touch each other) to a distance of $50 \mathrm{~mm}$, approximately 4 times the pore diameter. At large 
distances, the shape anisotropy of each individual pore is expected to dominate, whereas the effect of distribution anisotropy would become stronger with decreasing inter-pore distance. Spherical pores possess no shape anisotropy, so that the entire anisotropy observed in arrangements of spherical pores would be a direct consequence of distribution anisotropy. Arrangements of oblate and prolate ellipsoids allow to quantify the effect of each, shape and distribution anisotropy, as a function of pore distance. To investigate the influence of the intrinsic susceptibility, models were calculated for fluid susceptibilities of 4.0 (undiluted ferrofluid), 1.09 (as in Jones et al. [2006]), and 0.1 SI.

A second set of models was computed for the distribution anisotropy of the bedding-, capillary- and crack-like fabrics measured by Jones et al. [2006], with an intrinsic ferrofluid susceptibility of 1.09 SI. The bedding-like fabric was approximated by prolate pores aligned with $\mathrm{x}$. Distances between pores are not known, but constrained by pore and sample size to the range 2.0 (i.e. pores touching each other) to $5.8 \mathrm{~mm}$ (outer pores touching sample surface). The capillary-like fabric was modeled as prolate pores arranged in the xy plane, with distances between 3.3 and $5.5 \mathrm{~mm}$. The crack-like fabric was approximated by oblate pores forming a z-line, at distances between 1.4 and $6.8 \mathrm{~mm}$.

\section{Results}

4.1 Modeling results for single pores

Rotationally prolate ellipsoidal pores lead to prolate MPF ( $U=-1, L>1, F=1)$, and rotationally oblate pores to oblate $\operatorname{MPF}\left(U=1, L=1, F>1\right.$ ), with $k_{1}$ and $k_{3}$, respectively, parallel the unique axis (Figure 4a). Stronger pore anisotropy leads to larger degrees of anisotropy. The spherical pore possesses no anisotropy. These shape anisotropy models agree well with measurements on quasi-ellipsoidal pores, both in terms of fabric orientation and magnetic lineation and foliation [Jones et al., 2006].

The expected anisotropy of cylindrical voids was calculated in two ways:

approximating the cylinder by an ellipsoid with major and minor axis corresponding to length and diameter, and calculating the exact $N$ of the ellipsoid, or approximating $N$ for the cylinder shape. The modeled MPF of the near-isometric cylindrical void in Jones et al. [2006] matches their data better for the cylindrical approximation (Figure 4a). For the more elongated or flat cylinders forming parts of assemblies, both approximations result in similar predicted anisotropies. However, these single pore shape anisotropy models do not match the measurements (Figure 4b). A single flat cylinder displays a stronger foliation $(\mathrm{F}=1.8-1.9)$ compared to a stack of flat cylinders $(\mathrm{F}=1.4)$. Similarly, a single elongated cylinder has a stronger lineation $(\mathrm{L}>1.4)$ than a capillary-like arrangement of cylinders $(\mathrm{L} \sim 1.3)$. A bedding-like arrangement of cylinders displays both $\mathrm{F}$ and $\mathrm{L}$ of around 1.2, whereas a single cylinder component is prolate with $F=1$. These results clearly indicate that shape anisotropy alone cannot explain MPF of pore assemblies, and that distribution anisotropy needs to be taken into account as well.

Because Pfleiderer and Halls [1990] did not specify the susceptibility of their ferrofluid, the variation of modeled MPF with $k_{i n t}$ was investigated for their pore shapes as well as the spheroidal pores of Jones et al. [2006]. The latter allowed to explore the influence of $k_{\text {int }}$, whose modeled AMS fits well with measurements when the correct susceptibility is used. From these models, it is evident that intrinsic susceptibility has a major effect on the anisotropy parameters. For any non-isotropic shape, the degree of anisotropy increases when susceptibility is higher (Figure 5).

Pfleiderer and Halls [1990] measured 3 types of void shapes: rectangular prisms, cylinders, and an ellipsoidal cylinder. The expected MPF of the rectangular prisms, 
approximated by either oblate ellipsoids or rectangular rods, is oblate $(U=1, L=1, F>1)$ with $k_{3}$ along the unique axis. Both $\mathrm{P}$ and $\mathrm{F}$ increase with $k_{\text {int }}$ (Figure 6). The calculated MPF of the cylinders is prolate $\left(U=-1, L>1, F=1\right.$ ) with $k_{l}$ parallel to the cylinder axis, and also increases with intrinsic susceptibility. The models for the elliptical cylinder show that not only the degree of anisotropy, but also anisotropy shape varies with the intrinsic susceptibility of the fluid. A comparison of the models with the measurements by Pfleiderer and Halls [1990] indicates that the intrinsic susceptibility of their fluid was between 0.5 and 1 (SI).

\subsection{Shape and distribution anisotropy models for pore assemblies}

Models of spherical, oblate and prolate pores arranged in infinite lines and planes show the interplay between shape anisotropy and distribution anisotropy (Figures 7,8). At large distances, shape anisotropy dominates, and distribution anisotropy becomes increasingly important when pores get closer. Spherical pores arranged in lines display a prolate anisotropy with $k_{1}$ parallel to the line, whose anisotropy degree decreases with increasing distance. Planar arrangements of spherical pores possess oblate anisotropy with $k_{3}$ normal to the plane, decreasing again with increasing spacing between pores. Due to the absence of shape anisotropy, the fabric orientation is controlled entirely by the pore arrangement. The degree and shape of the total MPF are independent of the orientation of the lines or planes.

Oblate and prolate pores have uniaxial symmetry, where $\mathrm{z}$ is the unique axis. Therefore, the orientation of linear and planar arrangements plays an important role in defining the total MPF, consisting of a superposition of shape and distribution anisotropy contributing in different proportions (Figure 8). Linear arrangements parallel to $\mathrm{x}$ and $\mathrm{y}$ (and any direction within the symmetry plane of the pore) give rise to the same P, L, F and U, which are different from that of pores aligned along z. Similarly, planar assemblies in the yz and zx planes (and any plane perpendicular to the xy plane) behave similarly, and different from the planar arrangement in xy. Independent of this, and similar to arrangements of spherical pores, at close spacing, linear assemblies display prolate anisotropy with $k_{1}$ parallel to the line, and planar assemblies result in oblate anisotropy with $k_{3}$ normal to the plane.

Shape anisotropy dominates at large distances, with $k_{1}$, and $k_{3}$ parallel to $\mathrm{z}$ for prolate and oblate pores, respectively. Hence, principal axes directions are independent of pore spacing for prolate pores aligned with $\mathrm{z}$, or oblate pores arranged in the xy plane. For these configurations, the degree of anisotropy increases with decreasing pore spacing, and the shape is rotationally prolate $(U=-1)$ or rotationally oblate $(U=1)$ independent of pore spacing. Conversely, the shape changes, from $U=-1$ to $U=1$ and vice versa, at intermediate distances for oblate pores aligned with $\mathrm{z}$, and prolate pores arranged within the xy plane. With increasing pore spacing, the anisotropy degree decreases until the switch of axes accompanied by changes in shape, followed by an increase in anisotropy degree. For oblate pores aligned with $\mathrm{x}$ or $\mathrm{y}, P$ decreases, and $U$ changes gradually from -1 to 1 with increasing pore spacing. Analogously, planar arrangements of prolate pores in yz or zx planes, possess largest anisotropy when the pores are close, and decreasing $P$, accompanied with a gradual change from oblate to prolate shapes at increasing spacing. The most complex patterns are observed for oblate pores in yz or zx planes, or prolate pores arranged parallel to x or y. Due to a switch of principal axes directions, the observed anisotropy degree decreases and then remains constant, while the shape changes gradually from oblate to prolate to oblate, or from prolate to oblate to prolate.

The intrinsic susceptibility of the ferrofluid strongly affects the anisotropy degree $P$, magnetic lineation $L$, and magnetic foliation $F$; the stronger $k_{\text {int }}$, the higher these anisotropy parameters for a given pore configuration (Figure 9). However, the distance at which the 
behavior of the parameters (increasing, decreasing or constant) changes, appears independent of $k_{\text {int }}$. Also the principal directions and the parameter $U$ are largely independent of $k_{\text {int }}$.

Hence, the ferrofluid concentration affects anisotropy degree, but does not affect the distance at which either shape or distribution anisotropy are dominant. Thus, the values of $\mathrm{P}, \mathrm{F}$ and $\mathrm{L}$ do not uniquely define the pore fabric, but the same parameters can characterize different pore geometries measured with different ferrofluid susceptibility.

Models for combined shape and distribution anisotropy of the bedding-, capillary- and crack-like fabric of Jones et al. [2006] are shown in Figure 10. Modeled MPF parameters depend on the exact pore spacing, and a range of models are given for realistic spacings. The prolate shape anisotropy of the cylinders forming the bedding-like fabric turns into oblate and back to prolate with a $90^{\circ}$ rotation of the $k_{l}$ direction, as distribution anisotropy becomes more important. In other words, $L$ decreases while $F$ increases, until $L=1$, followed by increasing $L$ at constant $F$. Although it does not reproduce the measured data exactly, the modeled anisotropy possesses both lineation and foliation for a range of pore spacings (Figure 10a). For the capillary-like fabric, the effect of distribution anisotropy is a decrease in $L$ (while $F=1$ ), followed by an increase in $F$ (while $L=1$ ). The modeled superposition of shape and distribution anisotropy includes the measured MPF of Jones et al. [2006] (Figure 10b). The effect of distribution anisotropy on z-line arrangements of oblate pores is to decrease $F$ (at $L=1$ ) until $F=1$, then increasing $L$. Hence, it is likely that the $F$-value of the crack-like fabric is lower than that of the single pore shape anisotropy, as measured by Jones et al. [2006]. The MPF model for this configuration yields unrealistic negative magnetizations parallel to $\mathrm{x}$ and $\mathrm{y}$ for distances $<3.3 \mathrm{~mm}$, and very small positive magnetizations with unrealistically high P-values for distances of 3.4 to $3.6 \mathrm{~mm}$. These are likely artefacts related to the underlying assumption that the magnetization and secondary fields in each pore can be treated as uniform, which is only true as long as pores are small compared to their spacing. More sophisticated models would be needed to describe the secondary fields and magnetizations of large pores at small distances. For the purpose of this study, the models for distances $>3.6 \mathrm{~mm}$ will be used (Figure 10c).

\section{Discussion}

Magnetic fabrics are a powerful tool to characterize preferred mineral alignment in rocks, with a long history of applications in structural and tectonic studies [Borradaile and Henry, 1997; Borradaile and Jackson, 2010; Hrouda, 1982; Martín-Hernández et al., 2004; Owens and Bamford, 1976; Tarling and Hrouda, 1993]. There, magnetic fabrics serve as time- and cost-efficient measure for mineral texture, with the additional advantages that all grains are included independent of their size, a large and representative volume is measured, and the full anisotropy is obtained without the need for any a priori information on the fabric orientation. To adapt these advantages to the study of pore fabrics, Pfleiderer and Halls [1990] proposed the magnetic pore fabric method. Currently, the size range of pores that can be impregnated with ferrofluid and hence analysed by MPF, is limited to pores and pore throats $>10 \mathrm{~nm}$, the diameter of the nanoparticles in the ferrofluid [Parés et al., 2016]. In the future, different fluids may allow to investigate smaller pores. Models similar to those presented here can help estimate what susceptibilities are needed for such fluids to lead to a large enough anisotropy that can be measured reliably. Empirical relationships indicate large potential of the MPF method in predicting pore shape as well as permeability anisotropy and preferred flow directions. However, the large spread in empirical data has made the interpretation of pore fabrics from magnetic anisotropy data challenging.

Similar to the importance of carrier minerals for the interpretation of magnetic fabrics in rocks [Biedermann et al., 2018; Borradaile, 1987; Housen and van der Pluijm, 1990; Rochette, 1988; Rochette et al., 1992], a robust interpretation of MPF can only be performed 
once the origins of the pore magnetic anisotropy are understood in detail. MPFs have been attributed to the individual pore shape, and shape preferred orientation of multiple pores [Hrouda et al., 2000; Pfleiderer and Halls, 1990]. The equivalent pore concept [Hrouda et al., 2000] states that the AMS data, reflecting the demagnetization ellipsoid, quantifies the average pore shape. The models developed in the present study confirm that the MPF of a single pore is due to shape anisotropy, as proposed in the EPC theory. Further, the models show that the MPF not only depends on the pore geometry, but also on the intrinsic susceptibility (i.e., concentration) of the ferrofluid. This agrees with results observed by Jones et al. [2006] who found that for a single prolate pore, though always underestimating the physical anisotropy, the MPF lineation increases with ferrofluid concentration.

Shape anisotropy correctly predicts the MPF of single pores. However, in the case of pore assemblies, shape anisotropy alone fails to predict the MPF. A better agreement with experimental data is achieved by models that consider both shape and distribution anisotropy associated with groups of pores. The interaction between the two types of anisotropy is complex and depends on a number of factors, including (1) the shape of the individual pores, (2) the arrangement of pores relative to each other and the spacing between them, and (3) the intrinsic susceptibility of the ferrofluid. Not all previous MPF studies reported the susceptibility or concentration of the ferrofluid, but it is likely that differences in intrinsic susceptibility explain the variability in empirical relationships between anisotropy degree and pore shape or permeability anisotropy observed by different authors. More work is needed to understand the influence of pore throats and different pore sizes on the measured MPF, and to test how a large connected 3D network of ferrofluid compares to single pores interacting with each other. On the microscale, ferrofluid consists of magnetic nanoparticles suspended in water or oil. Thus, it needs to be investigated whether the ferrofluid acts as a homogeneous fluid throughout the pore space of a rock, or if the particles are concentrated at the grain-pore interface, or cluster around certain minerals, e.g. minerals with specific wettability or magnetic properties. Different minerals have different wettability [Abdallah et al., 2007], so that ferrofluid may not fill all pore space equally, and impregnation with water-based and oilbased ferrofluid may result in different MPFs. It is also possible that the magnetic particles in the fluid aggregate around magnetite grains in the rock, leading to non-homogeneous

impregnation. The effects of these on the MPF and related pore fabric interpretation needs to be characterized in detail.

Despite the number of open questions, the present study is an important step towards a better understanding of the factors defining MPFs. It may explain some of the variability observed in empirical studies correlating MPF with pore shape or permeability anisotropy, in that it shows the importance of pore distribution in addition to shape in defining the fabric. In addition to pore fabric geometry, the intrinsic susceptibility of the ferrofluid plays a major role in defining the anisotropy parameters $P, L$ and $F$.

\section{Conclusions}

The magnetic pore fabric method has great potential to help analyse pore fabrics in $3 \mathrm{D}$, covering a large number of pores down to sizes of $10 \mathrm{~nm}$. This study shows, that models taking into account shape anisotropy and distribution anisotropy can reliably predict the MPF of samples with known pore shapes that had been measured in previous studies [Jones et al., 2006; Pfleiderer and Halls, 1990]. The models help characterize how the intrinsic susceptibility of the ferrofluid affect the measured anisotropy parameters $P, L$ and $F$, which may explain some of the variability in existing empirical relationships. Work is still needed to describe all factors contributing to MPF in full detail. Nevertheless, this study provides an important step towards a better understanding of MPF and a robust and reliable interpretation of pore fabrics from magnetic data. 


\section{Acknowledgments}

Joshua Feinberg and Mike Jackson are gratefully acknowledged for their comments on a previous version of this manuscript. Josep M Pares and Philip Benson are thanked for their constructive reviews. This study was funded by the Swiss National Science Foundation, project 176917. Modeling results are available in the manuscript, and experimental results are published in the cited literature.

\section{References}

Abbott, J. J., O. Ergeneman, M. P. Kummer, A. M. Hirt, and B. J. Nelson (2007), Modeling magnetic torque and force for controlled manipulation of soft-magnetic bodies, IEEE Transactions on Robotics, 23(6), 1247-1252.

Abdallah, W., et al. (2007), Fundamentals of Wettability, Oilfield Review, 19(2), 44-61.

Almqvist, B. S. G., D. Mainprice, C. Madonna, L. Burlini, and A. M. Hirt (2011), Application of differential effective medium, magnetic pore fabric analysis, and X-ray microtomography to calculate elastic properties of porous and anisotropic rock aggregates, Journal of Geophysical Research-Solid Earth, 116, doi: 10.1029/2010jb007750.

Archanjo, C. J., P. Launeau, and J. L. Bouchez (1995), Magnetic fabric vs. magnetite and biotite shape fabrics of the magnetite-bearing granite pluton of Gameleiras (Northeast Brazil), Physics of the Earth and Planetary Interiors, 89, 63-75.

Balsley, J. R., and A. F. Buddington (1960), Magnetic susceptibility anisotropy and fabric of some Adirondack granites and orthogneisses, American Journal of Science, 258-A, 620.

Bear, J. (2013), Dynamics of Fluids in Porous Media, Dover Publications Inc, New York, USA.

Bear, J., C. Braester, and P. C. Menier (1987), Effective and relative permeabilities of anisotropic porous media, Transport in Porous Media, 2(3), 301-316.

Beard, D. C., and P. K. Weyl (1973), Influence of texture on porosity and permeability of unconsolidated sand, AAPG Bulletin, 57, 349-369.

Bennett, R. H., K. M. Fischer, D. L. Lavoie, W. R. Bryant, and R. Rezak (1989), Porometry and fabric of marine clay and carbonate sediments: Determinants of permeability, Marine Geology, 89(1-2), 1277-1152, doi: 10.1016/0025-3227(89)90030-3.

Benson, P. M., P. G. Meredith, and E. S. Platzman (2003), Relating pore fabric geometry to acoustic and permeability anisotropy in Crab Orchard Sandstone: A laboratory study using magnetic ferrofluid, Geophysical Research Letters, 30(19), 1976.

Biedermann, A. R. (2018), Magnetic Anisotropy in Single Crystals: A Review, Geosciences, 8(8), doi: 10.3390/geosciences8080302.

Biedermann, A. R., K. Kunze, and A. M. Hirt (2018), Interpreting magnetic fabrics in amphibole-bearing rocks, Tectonophysics, 722, 566-576.

Borradaile, G. (1987), Anisotropy of magnetic susceptibility: rock composition versus strain, Tectonophysics, 138, 327-329.

Borradaile, G. J., and B. Henry (1997), Tectonic applications of magnetic susceptibility and its anisotropy, Earth-Science Reviews, 42, 49-93.

Borradaile, G. J., and M. Jackson (2010), Structural geology, petrofabrics and magnetic fabrics (AMS, AARM, AIRM), Journal of Structural Geology, 32(10), 1519-1551, doi: 10.1016/j.jsg.2009.09.006.

Borradaile, G. J., R. A. Stewart, and T. Werner (1993), Archean uplift of a subprovince boundary in the Canadian Shield, revealed by magnetic fabrics, Tectonophysics, 227(14), 1-15, doi: 10.1016/0040-1951(93)90083-v. 
Cañón-Tapia, E. (1996), Single-grain versus distribution anisotropy: a simple threedimensional model, Physics of the Earth and Planetary Interiors, 94, 149-158.

Cañón-Tapia, E. (2001), Factors affecting the relative importance of shape and distribution anisotropy in rocks: theory and experiments, Tectonophysics, 340, 117-131.

Clark, D. A. (2014), Methods for determining remanent and total magnetizations of magnetic sources - a review, Exploration Geophysics, 45, 271-304.

Clark, D. A., and D. W. Emerson (1999), Self-Demagnetisation, Preview, 79, 22-25.

Dullien, F. A. L. (1992), Porous Media: Fluid Transport and Pore Structure, 2nd ed., Academic Press Inc, San Diego, USA.

Esteban, L., Y. Géraud, and J. L. Bouchez (2006), Pore network geometry in low permeability argillites from magnetic fabric data and oriented mercury injections, Geophysical Research Letters, 33, L18311, doi: 10.1029/2006GL026908.

Gaillot, P., M. de Saint-Blanquat, and J.-L. Bouchez (2006), Effects of magnetic interactions in anisotropy of magnetic susceptibility: Models, experiments and implications for igneous rock fabrics quantification, Tectonophysics, 418, 3-19.

Graham, J. W. (1954), Magnetic susceptibility anisotropy, an unexploited petrofabric element, Geological Society of America Bulletin, 65(12), 1257-1258.

Grégoire, V., M. De Saint-Blanquat, A. Nédélec, and J.-L. Bouchez (1995), Shape anisotropy versus magnetic interactions of magnetite grains: experiments and application to AMS in granitic rocks, Geophysical Research Letters, 22(20), 2765-2768.

Grégoire, V., P. Darrozes, P. Gaillot, and A. Nédélec (1998), Magnetite grain shape fabric and distribution anisotropy vs rock magnetic fabric: a three-dimensional case study, Journal of Structural Geology, 20(7), 937-944.

Hailwood, E. A., D. Bowen, F. Ding, P. W. M. Corbett, and P. Whattler (1999), Characterizing pore fabrics in sediments by anisotropy of magnetic susceptibility analyses, in Paleomagnetism and Diagenesis in Sediments, edited by D. H. Tarling and P. Turner, pp. 125-126, Geological Society, London, Special Publications.

Hargraves, R. B. (1959), Magnetic anisotropy and remanent magnetism in hemo-ilmenite from ore deposits at Allard Lake, Quebec, Journal of Geophysical Research, 64(10), 1565-1578, doi: 10.1029/JZ064i010p01565.

Hargraves, R. B., D. Johnson, and C. Y. Chan (1991), Distribution anisotropy: the cause of AMS in igneous rocks?, Geophysical Research Letters, 18(12), 2193-2196.

Hirt, A. M., W. Lowrie, W. S. Clendenen, and R. Kligfield (1988), The correlation of magnetic anisotropy with strain in the Chelmsford Formation of the Sudbury Basin, Ontario, Tectonophysics, 145, 177-189.

Housen, B. A., and B. A. van der Pluijm (1990), Chlorite control of correlations between strain and anisotropy of magnetic susceptibility, Physics of the Earth and Planetary Interiors, 61, 315-323.

Hrouda, F. (1982), Magnetic anisotropy of rocks and its application in geology and geophysics, Geophysical Surveys, 5, 37-82.

Hrouda, F., J. Hanak, and I. Terzijski (2000), The magnetic and pore fabrics of extruded and pressed ceramic models, Geophysical Journal International, 142, 941-947.

Jelinek, V. (1981), Characterization of the magnetic fabric of rocks, Tectonophysics, 79, T63T67.

Jones, C., and P. Meredith (1998), An experimental study of elastic wave propagation anisotropy and permeability anisotropy in an illitic shale, in SPE/ISRM Rock Mechanics in Petroleum Engineering, edited, Society of Petroleum Engineers, Trondheim, Norway. 
Jones, S., P. Benson, and P. Meredith (2006), Pore fabric anisotropy: testing the equivalent pore concept using magnetic measurements on synthetic voids of known geometry, Geophysical Journal International, 166, 485-492.

Khan, M. A. (1962), The anisotropy of magnetic susceptibility of some igneous and metamorphic rocks, Journal of Geophysical Research, 67(7), 2873-2885.

Kligfield, R., W. Lowrie, and W. D. Dalziel (1977), Magnetic susceptibility anisotropy as a strain indicator in the Sudbury Basin, Ontario, Tectonophysics, 40, 287-308.

Kneen, S. J. (1976), The relationship between the magnetic and strain fabrics of some haematite-bearing slates, Earth and Planetary Science Letters, 31, 413-416.

Louis, L., C. David, V. Metz, P. Robion, B. Menendez, and C. Kissel (2005), Microstructural control on the anisotropy of elastic and transport properties in undeformed sandstones, International Journal of Rock Mechanics and Mining Sciences, 42, 911-923.

Lowrie, W. (1997), Fundamentals of Geophysics, 354 pp., Cambridge University Press, Cambridge, UK.

Mainprice, D., and M. Humbert (1994), Methods of calculating petrophysical properties from lattice preferred orientation data, Surveys in Geophysics, 15, 575-592.

Martín-Hernández, F., C. M. Lüneburg, C. Aubourg, and M. Jackson (2004), Magnetic Fabrics: Methods and Applications, The Geological Society, London, UK.

Muxworthy, A. R., and W. Williams (2004), Distribution anisotropy: the influence of magnetic interactions on the anisotropy of magnetic remanence, in Magnetic Fabric: Methods and Applications, edited by F. Martin-Hernandez, C. M. Lüneburg, C. Aubourg and M. Jackson, pp. 37-47, The Geological Society of London, London, UK.

Nabawy, B. S., P. Rochette, and Y. Géraud (2009), Petrophysical and magnetic pore network anisotropy of some cretaceous sandstone from Tushka Basin, Egypt, Geophysical Journal International, 177, 43-61.

Osborn, J.A. (1945), Demagnetizing factors of the general ellipsoid, Physical Review, 67(1112), 351-357, doi: 10.1103/PhysRev.67.351.

Owens, W. H., and D. Bamford (1976), Magnetic, seismic, and other anisotropic properties of rock fabrics, Philosophical Transactions of the Royal Society A, 283, 55-68.

Parés, J., L. Miguens, and C. Saiz (2016), Characterizing pore fabric in sandstones with magnetic anisotropy methods: initial results, Journal of Petroleum Science and Engineering, 143, 113-120.

Parry, L. G. (1965), Magnetic properties of dispersed magnetite powders, The Philosophical Magazine: A Journal of Theoretical Experimental and Applied Physics, 11(110), 303 312, doi: 10.1080/14786436508221858.

Pfleiderer, S., and H. C. Halls (1990), Magnetic susceptibility anisotropy of rocks saturated with ferrofluid: a new method to study pore fabric?, Physics of the Earth and Planetary Interiors, 65, 158-164.

Pfleiderer, S., and H. C. Halls (1993), Magnetic pore fabric analysis: Verification through image autocorrelation, Journal of Geophysical Research, 98(B3), 4311-4316.

Pfleiderer, S., and H. C. Halls (1994), Magnetic pore fabric analysis: a rapid method for estimating permeability anisotropy, Geophysical Journal International, 116, 39-45.

Rasolofosaon, P. N. J., and B. E. Zinszner (2002), Comparison between permeability anisotropy and elasticity anisotropy of reservoir rocks, Geophysics, 67, 230-240.

Robion, P., C. David, J. Dautriat, J.-C. Colombier, L. Zinsmeister, and P.-Y. Collin (2014), Pore fabric geometry inferred from magnetic and acoustic anisotropies in rocks with various mineralogy, permeability and porosity, Tectonophysics, 629, 109-122.

Rochette, P. (1988), Inverse magnetic fabric in carbonate-bearing rocks, Earth and Planetary Science Letters, 90, 229-237. 
Rochette, P., M. Jackson, and C. Aubourg (1992), Rock magnetism and the interpretation of anisotropy of magnetic susceptibility, Reviews of Geophysics, 30(3), 209-226.

Rochette, P., C. Aubourg, and M. Perrin (1999), Is this magnetic fabric normal? A review and case studies in volcanic formations, Tectonophysics, 307, 219-234.

Salazar, C. A., C. Bustamante, and C. J. Archanjo (2016), Magnetic fabric (AMS, AAR) of the Santa Marta batholith (northern Colombia) and the shear deformation along the Caribbean Plate margin, Journal of South American Earth Sciences, 70, 55-68, doi: 10.1016/j.jsames.2016.04.011.

Sato, M., and Y. Ishii (1989), Simple and approximate expressions of demagnetizing factors of uniformly magnetized rectangular rod and cylinder, Journal of Applied Physics, 66(2), 983-985.

Schön, J.H. (2015), Physical Properties of Rocks: Fundamentals and Principles of Petrophysics, 2nd ed., Elsevier, Amsterdam, Netherlands.

Stephenson, A. (1994), Distribution anisotropy: two simple models for magnetic lineation and foliation, Physics of the Earth and Planetary Interiors, 82, 49-53.

Stoner, E. C. (1945), The demagnetizing factors for ellipsoids, The London, Edinburgh, and Dublin Philosophical Magazine and Journal of Science: Series 7, 36(263), 803-821.

Tarling, D. H., and F. Hrouda (1993), The magnetic anisotropy of rocks, Chapman and Hall, London, UK.

Trindade, R. I. F., M. I. B. Raposo, M. Ernesto, and R. Siqueira (1999), Magnetic susceptibility and partial anhysteretic remanence anisotropies in the magnetite-bearing granite pluton of Tourao, NE Brazil, Tectonophysics, 314, 443-468.

Weger, R. J., G. P. Eberli, G. T. Baechle, J. L. Massaferro, and Y.-F. Sun (2009),

Quantification of pore structure and its effect on sonic velocity and permeability in carbonates, AAPG Bulletin, 93(10), 1297-1317.

Wing-Fatt, L., and F. D. Stacey (1966), Magnetic anisotropy of laboratory materials in which magma flow is simulated, Pure and Applied Geophysics, 64, 78-80. 


\section{Figure captions}
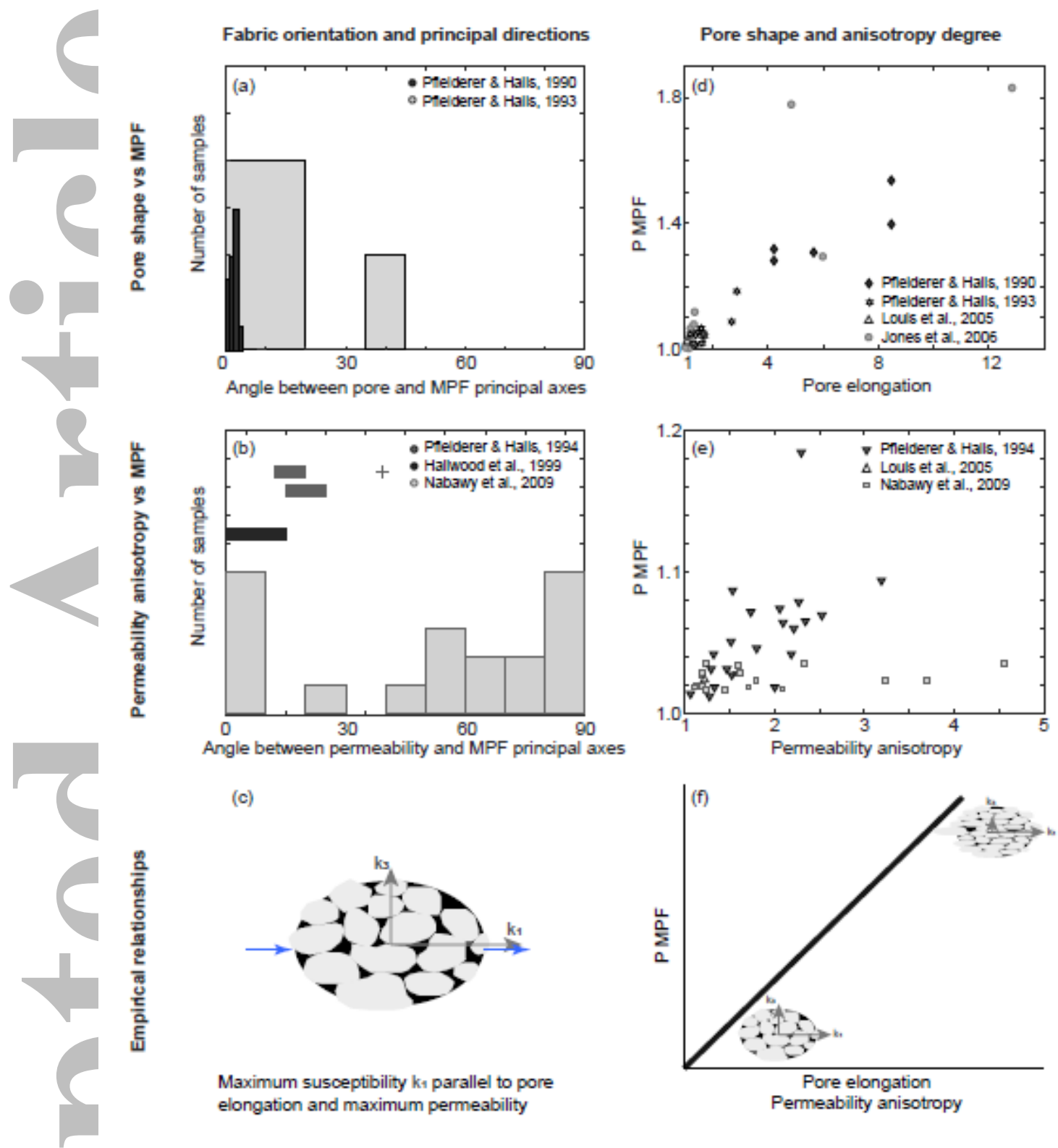

(c)
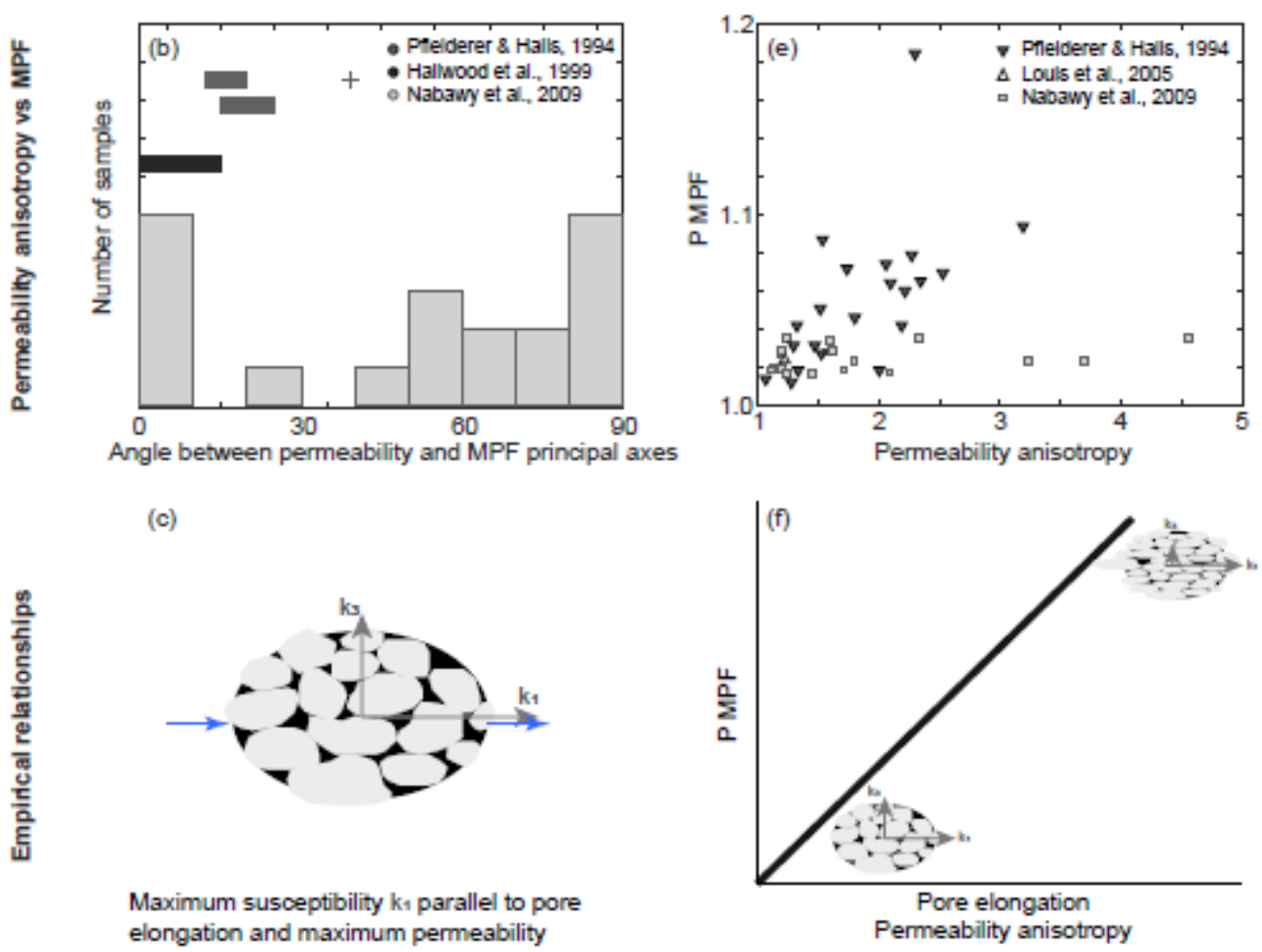

Figure 1: Compilation of data on the relationship between MPF and pore shape or permeability anisotropy for principal directions and anisotropy degree, and commonly used empirical relationships to interpret MPF for predicting pore fabrics and preferred flow directions. ( $a, b)$ Orientations of MPF principal axes with respect to pore shape or permeability anisotropy are presented as histograms for studies where individual specimen data was available, or observed ranges where results were summarized over all samples. (c) Empirical relationship derived from the data in $(\mathrm{a}, \mathrm{b})$. (d, e) Correlation between pore elongation (ratio of longest to shortest axis) or permeability anisotropy (ratio of highest to lowest measured permeability) to the $P$-value of the MPF fabric. $P$ is defined as the ratio of maximum principal susceptibility $k_{1}$ to minimum principal susceptibility $k_{3}$, i.e. $P=k_{1} / k_{3}$. (f) Empirical relationship derived from the data in (c, d). 
Pfleiderer \& Halls, 1990
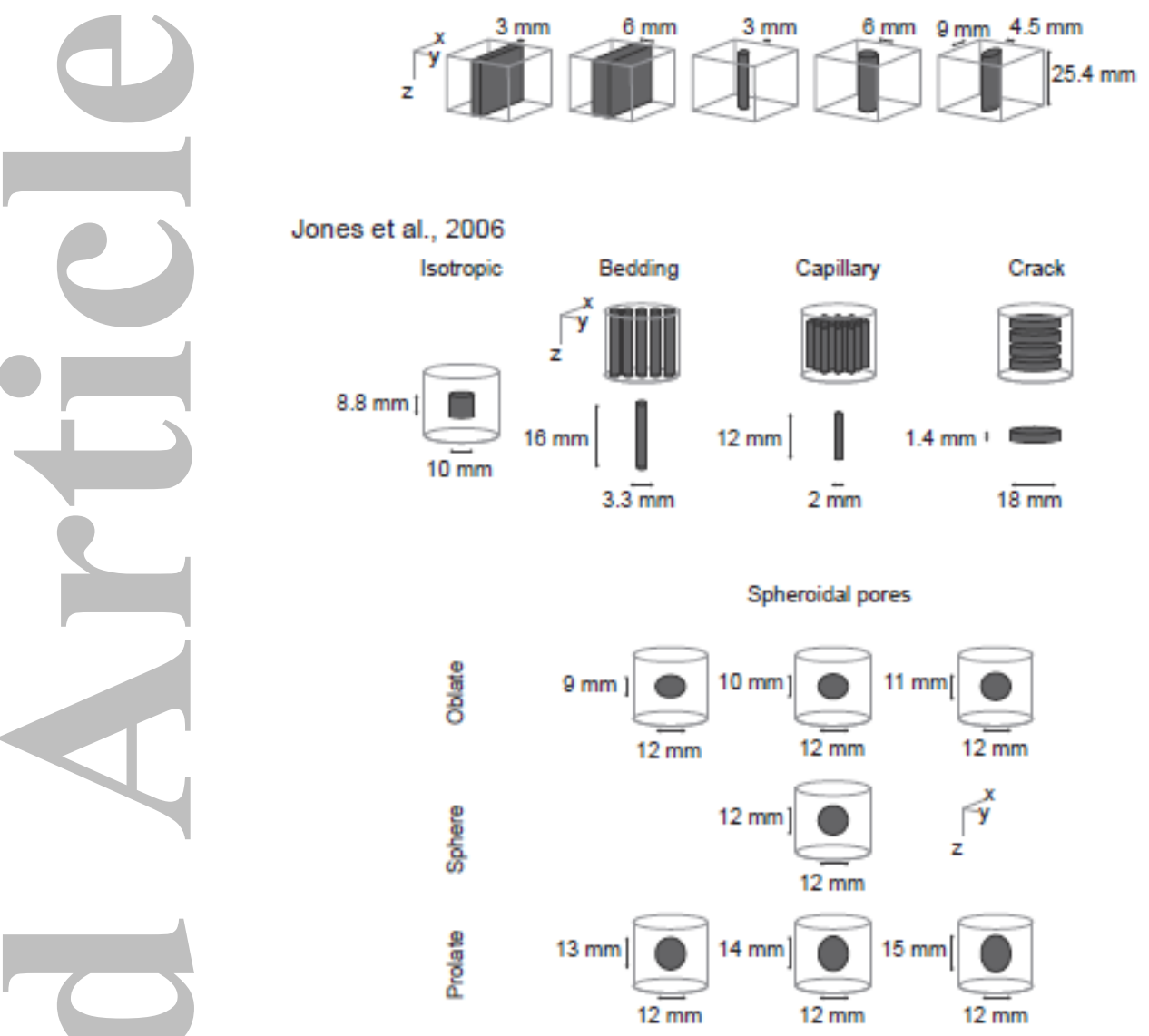

Figure 2: Simplified pore shapes, modified after Pfleiderer and Halls [1990], and Jones et al. [2006].

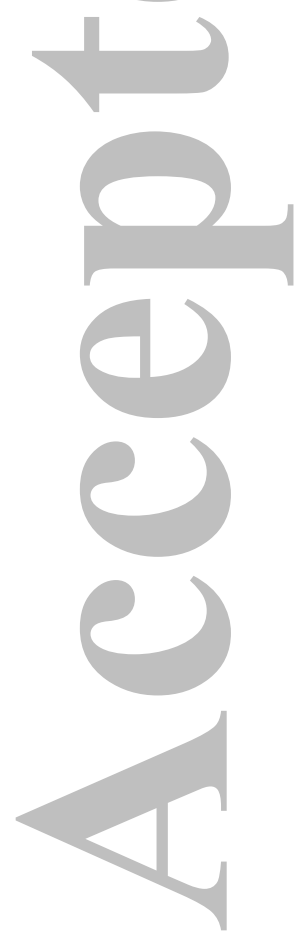



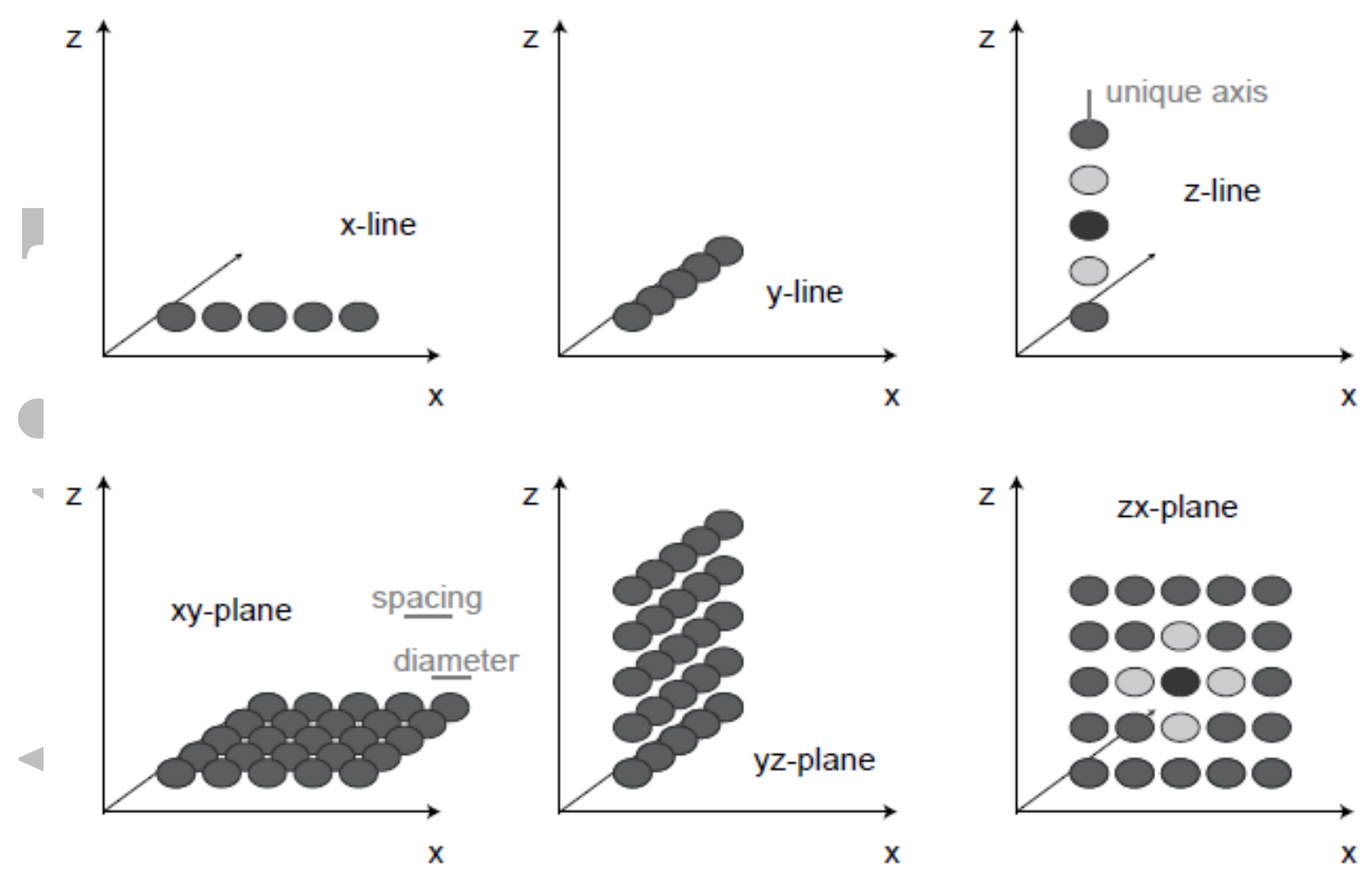

Figure 3: Pore arrangements. The model considers lines or planes of equal pores, at constant distance. Light grey pores indicate nearest neighbors taken into account for magnetostatic interactions with the middle pore. The unique axis for oblate and prolate pores (i.e., symmetry axis) is parallel to $\mathrm{z}$.
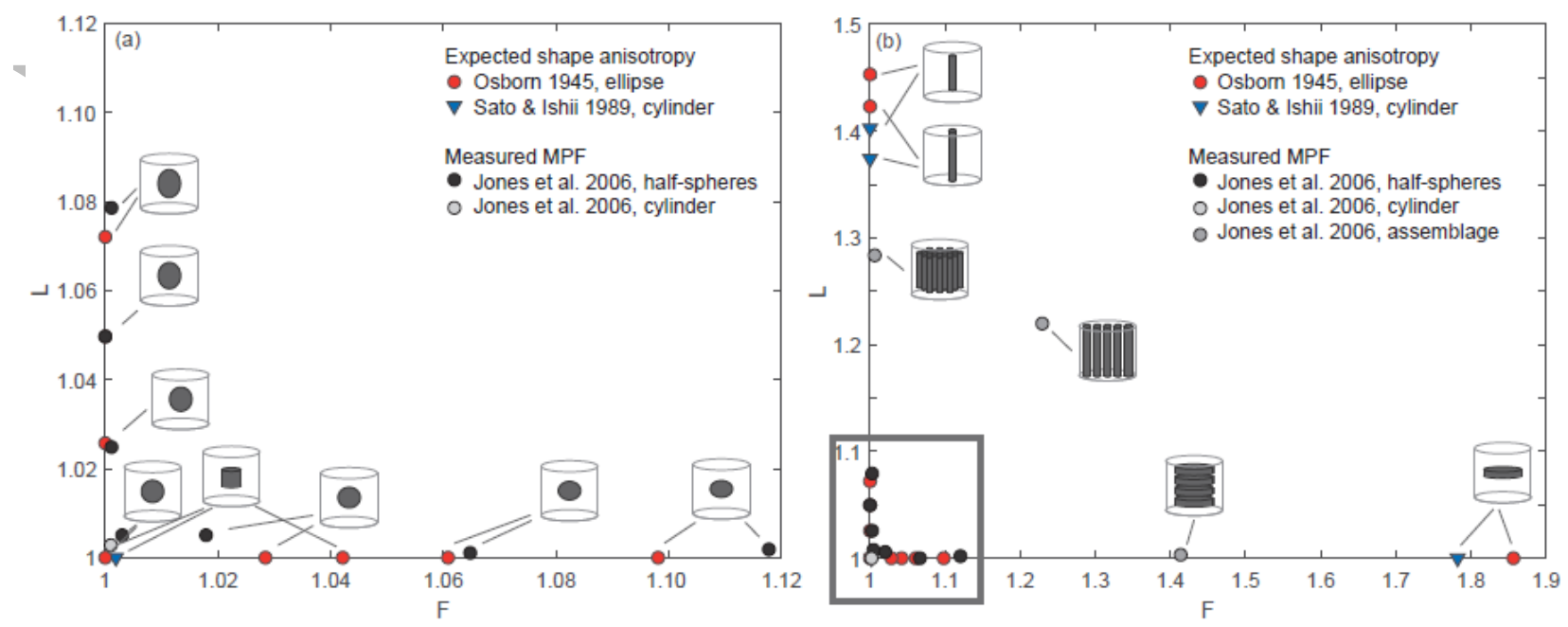

Figure 4: Magnetic anisotropy in synthetic samples with different pore shapes for single ellipsoidal pores (a), and arrangements of cylindrical pores (b). Comparison between shape anisotropy models and published data. 


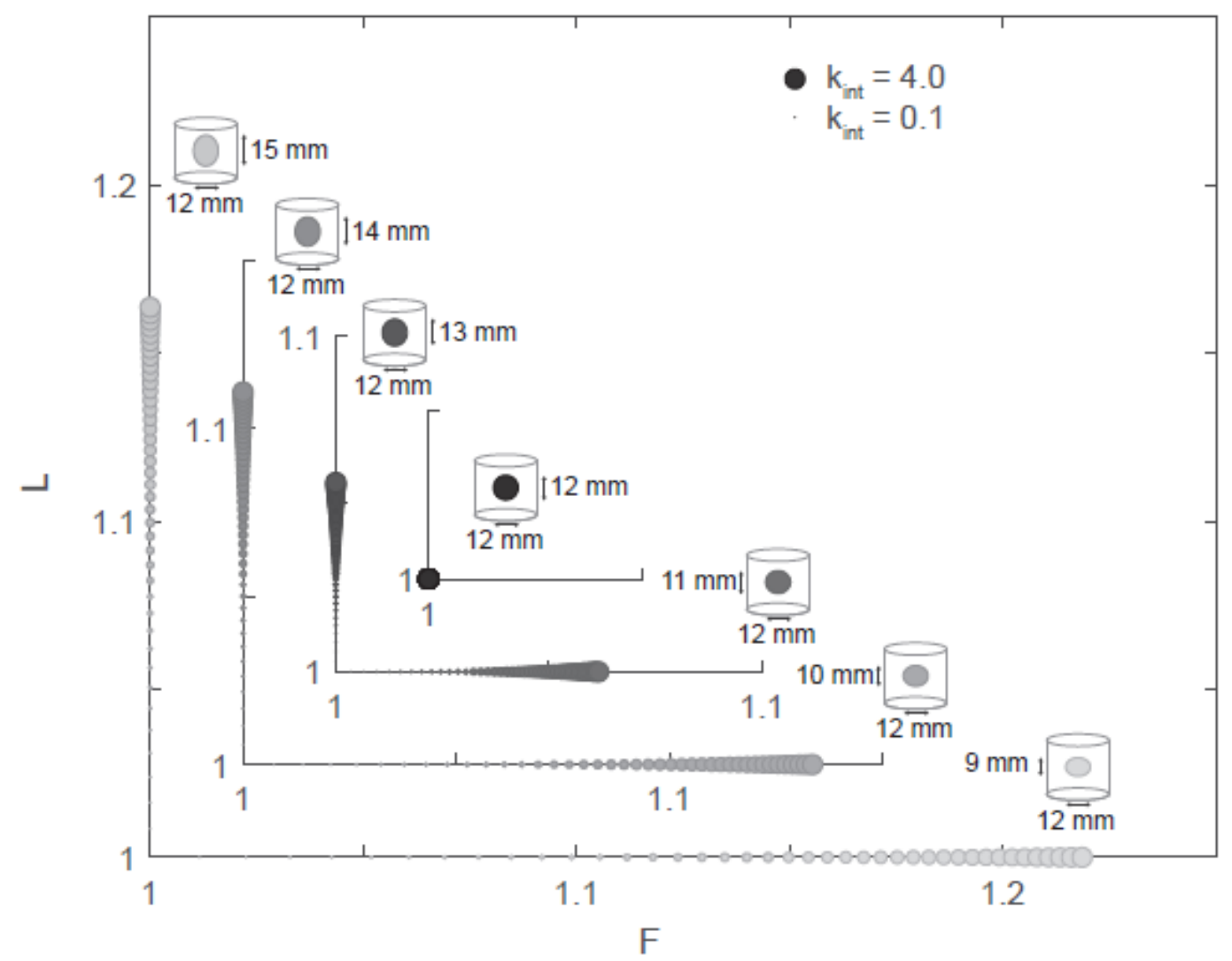

Figure 5: Influence of fluid susceptibility on measured MPF. One point for every 0.1 (SI) for susceptibilities in the range of 0.1 to 4.0 SI. The size of the point relates to the strength of the susceptibility of the fluid in the void. A larger anisotropy can be due to (1) stronger deviation from spherical shape, or (2) larger intrinsic susceptibility. 

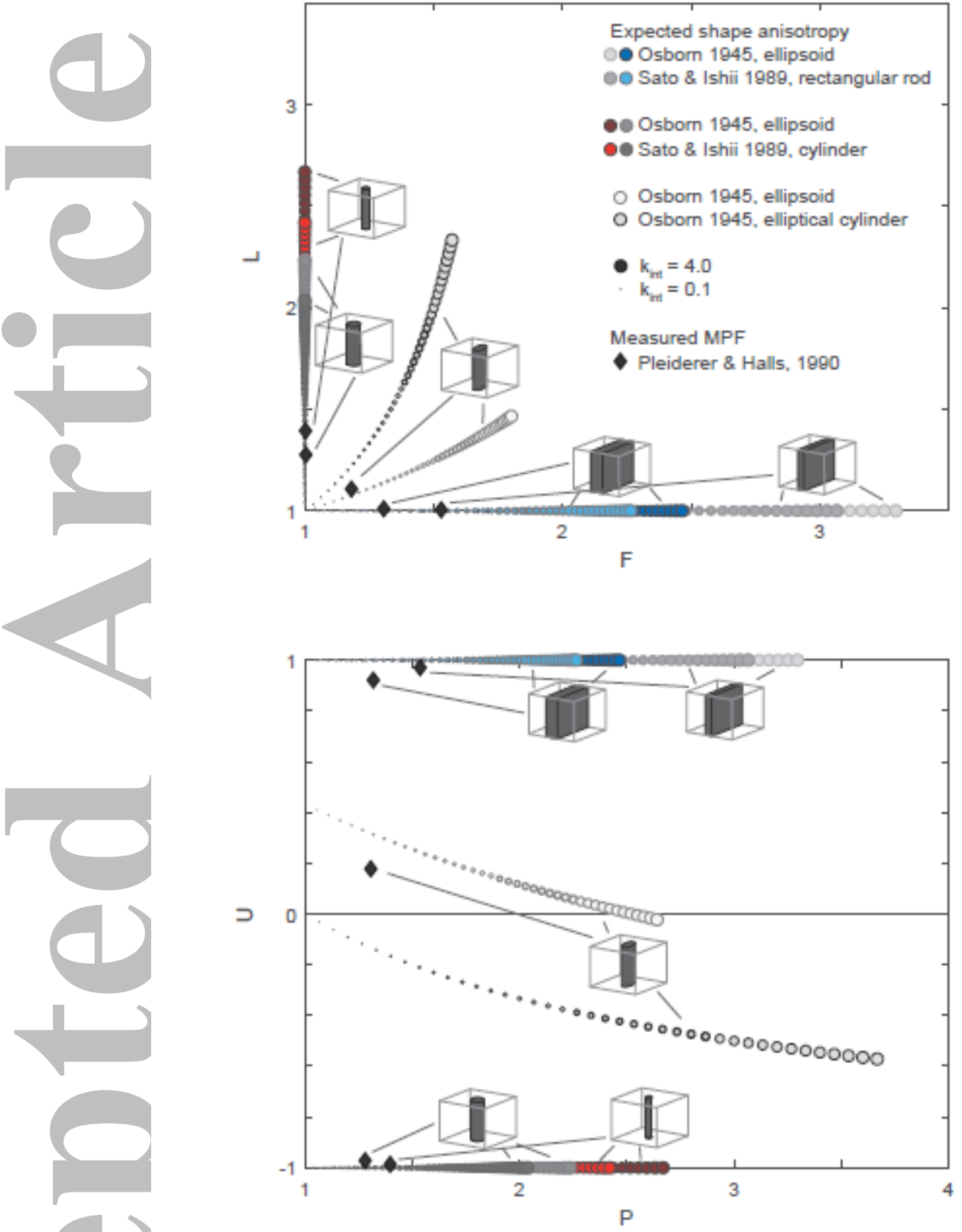

Figure 6: Models vs measurements for the voids of Pfleiderer \& Halls, 1990. A comparison between models and measurements indicates that their ferrofluid was diluted to an intrinsic susceptibility in the range of 0.5 to 1 . Note that not only anisotropy degree, but also anisotropy shape can vary with intrinsic susceptibility of the fluid. 


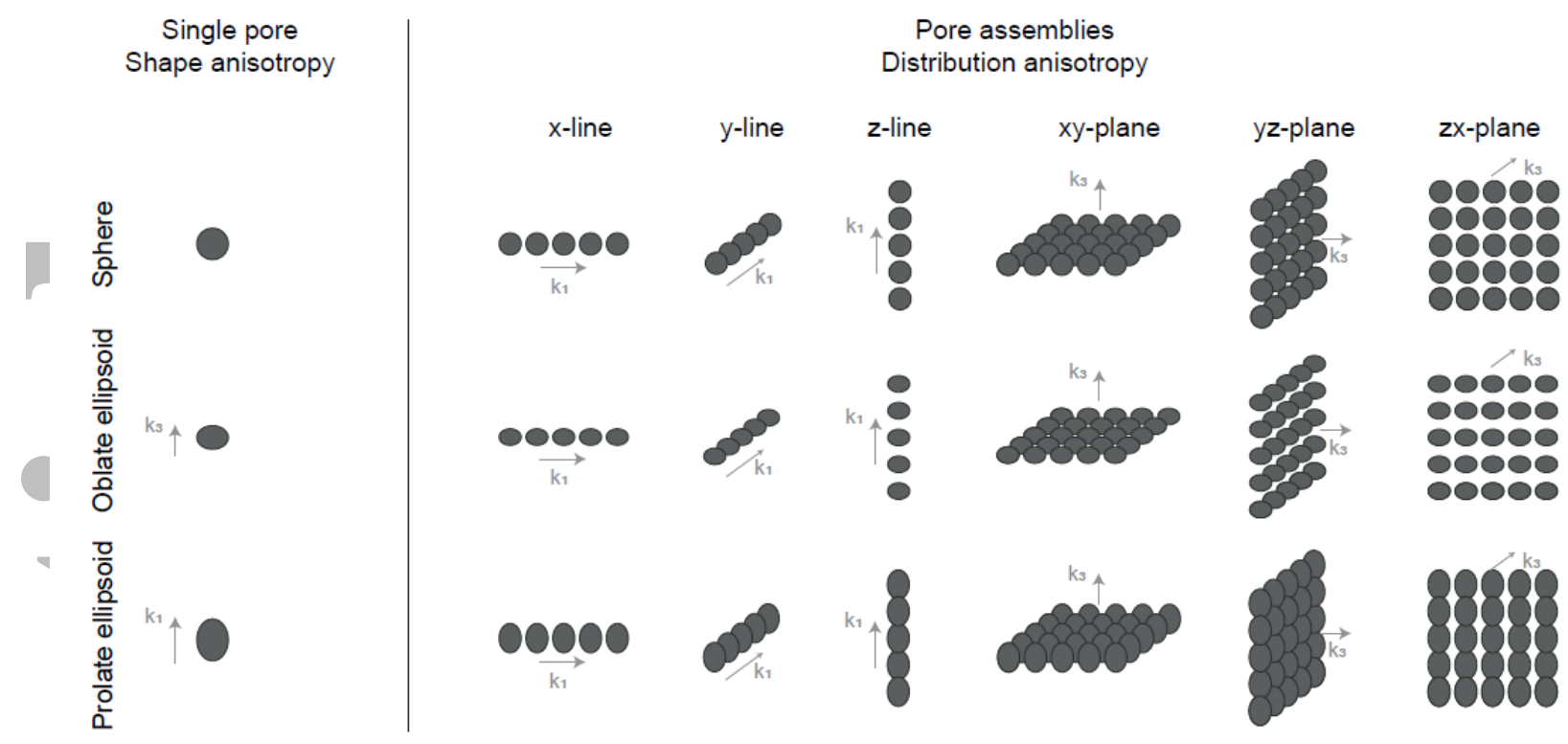

Figure 7: Principal axes directions for shape anisotropy and distribution anisotropy of spherical, oblate and prolate pores in linear and planar arrangements.

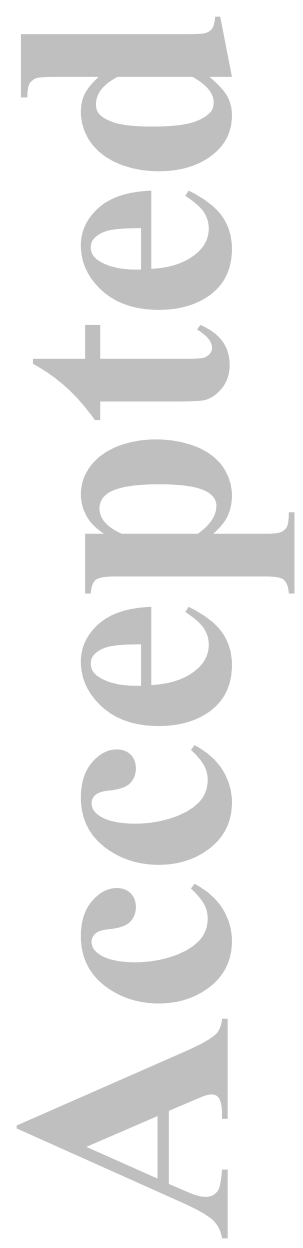




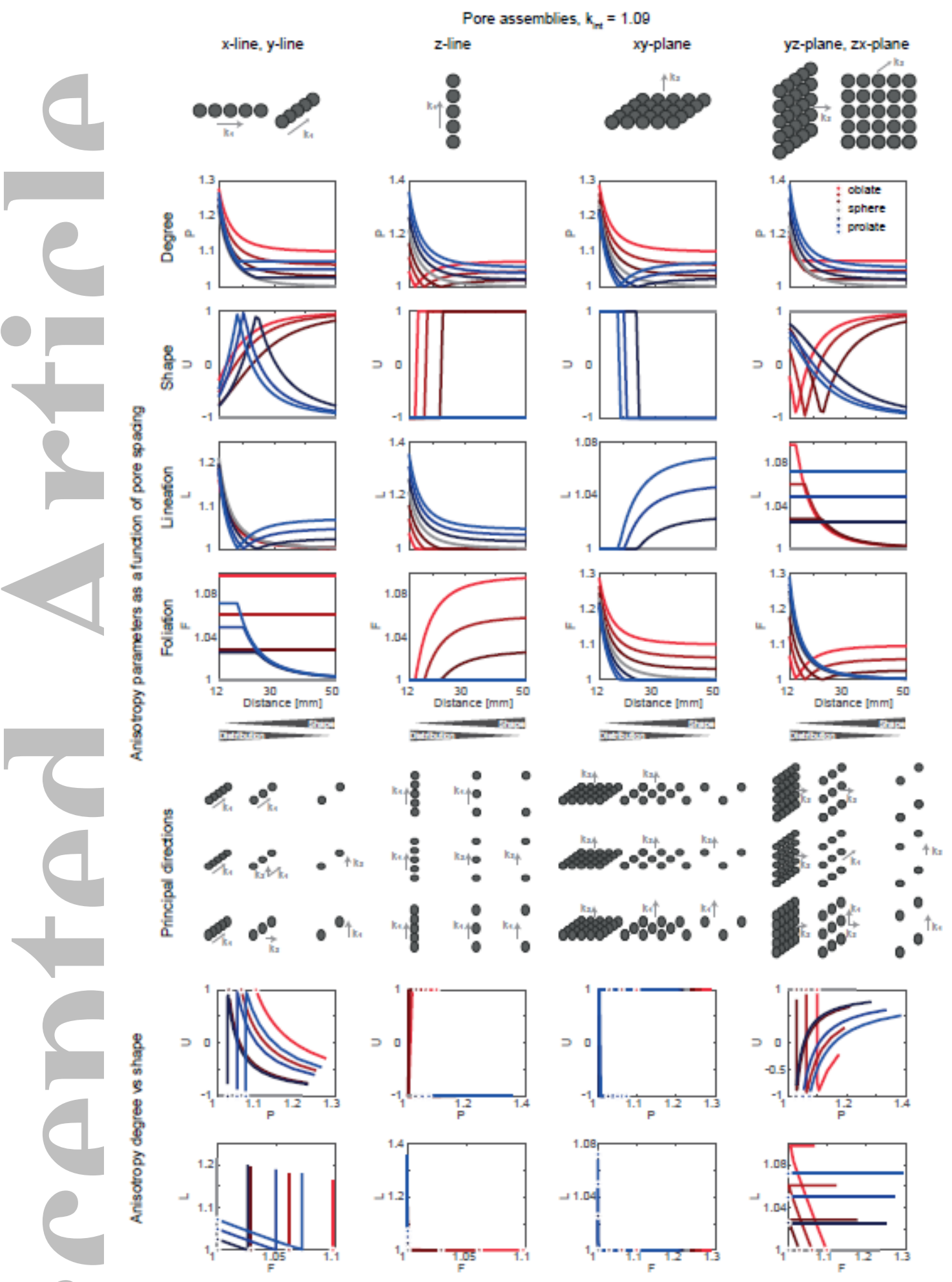

Figure 8: Changes in anisotropy parameters with pore spacing for spherical, oblate and prolate pores in linear or planar arrangements. Ellipsoidal pores have a radial diameter of 12 $\mathrm{mm}$, and axial diameters of $9-15 \mathrm{~mm}$. All parameters calculated for an intrinsic susceptibility of 1.09 (SI). Circles in the bottom rows indicate anisotropy parameters for a single pore of the same shape. 


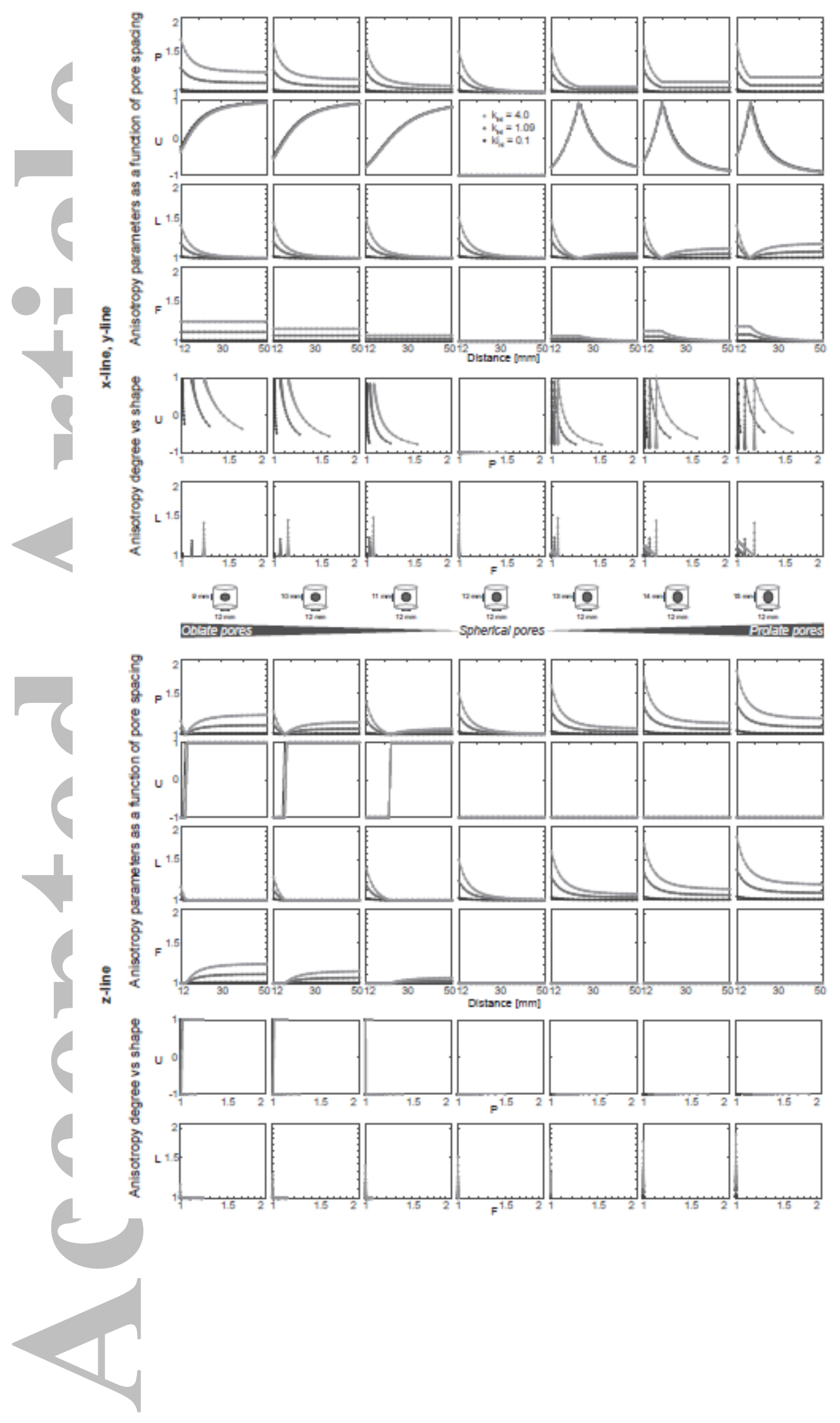

(C2019 American Geophysical Union. All rights reserved. 


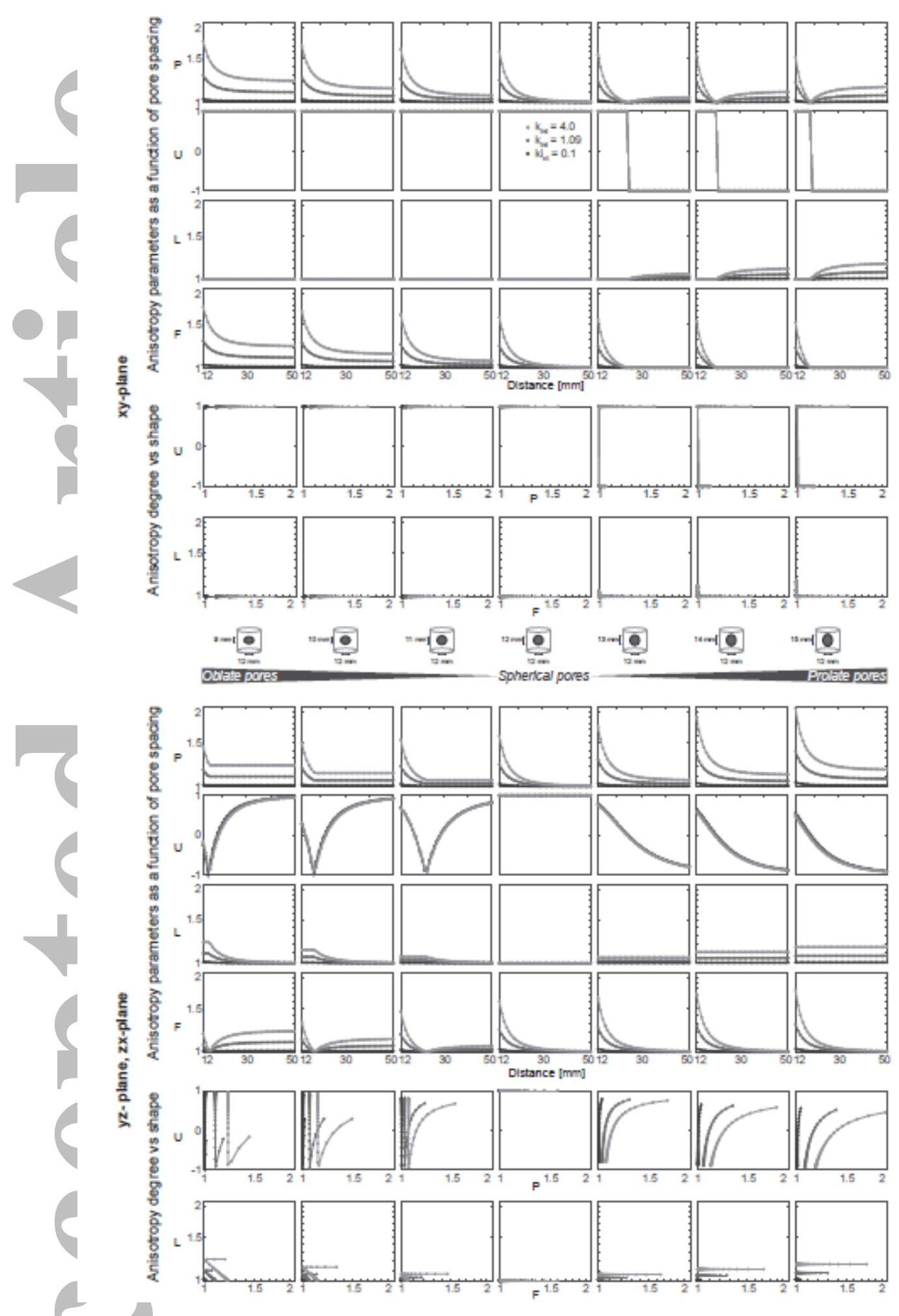

Figure 9: Variation of anisotropy parameters $(P, U, L, F)$ with distance between pores as a function of ferrofluid intrinsic susceptibility. Logarithmic scales were used for $P, L$, and $F$ to better visualize changes at small anisotropies. (a) Linear pore assemblies. (b) Planar pore assemblies 

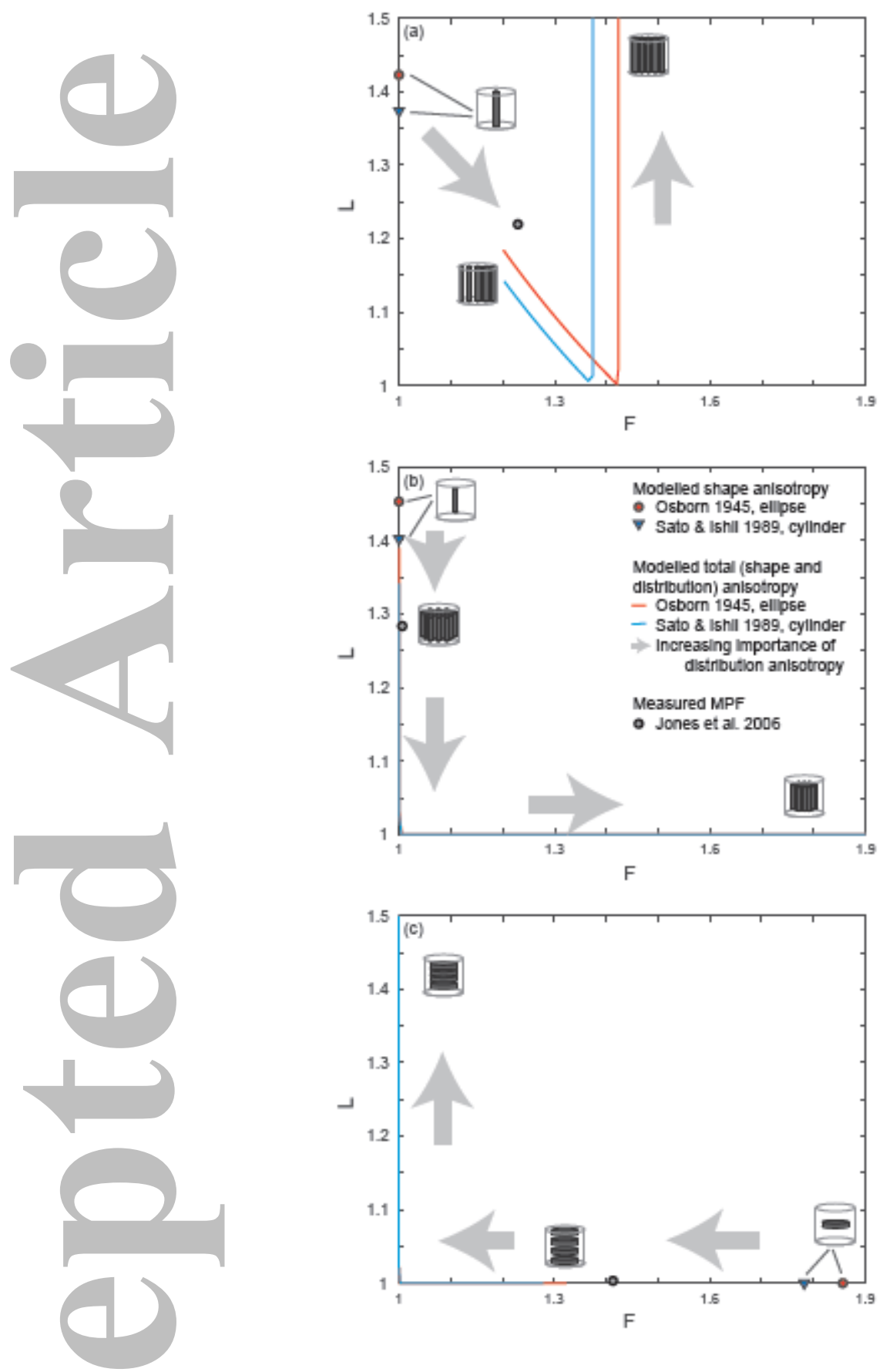

Figure 10: Modeled MPF for a combination of shape and distribution anisotropy. Grey arrows indicate the effect of increasing importance of distribution anisotropy, i.e. closer pore spacing. 